\title{
An Open Multicenter Study of the Use of Gatifloxacin for the Treatment of Non- Complicated Acute Bacterial Rhinosinusitis in Adults
}

Weckx L.L.M. ${ }^{1}$, Campos C.A.H. ${ }^{2}$, Sakano E. ${ }^{3}$, Pignatari S.S.N. ${ }^{4}$ and Figueiredo C.R. ${ }^{5}$

\author{
Departament of Otolaryngology- Federal University São \\ Paulo $^{\text {; }}$ Dept. Otorhinolaryngology- Santa Casa Misericórdia \\ Medical School São ${ }^{2}$; Dept. Otorhinolaryngology and Head \\ Neck Surgery University of Campinas ${ }^{3}$; Division of Pediatric \\ Otolaryngology- Federal University of São Paulo ${ }^{4}$; Fellow in \\ Pediatric Otorhinolaryngology - Division of Pediatric \\ Otolaryngology- Federal University of São Paulos.
}

\begin{abstract}
The bacteriological and clinical efficacy and the safety of gatifloxacin for the treatment of noncomplicated acute rhinosinusitis was evaluated in 49 adult patients in an open-label multicenter study in Brazil. Patients under age 18, or with associated systemic diseases, were excluded. Diagnosis was based on symptoms, otorhinolaryngological examination, and X-rays of the sinus. At the first visit, all patients were treated with a single daily dose of $\mathbf{4 0 0} \mathbf{~ m g}$ gatifloxacin for $\mathbf{1 0}$ days. Middle nasal meatus secretion was collected and sent for culture before and after treatment. Patients were all reevaluated at days 3 to 5 ; days +1 to +5 and 18 to 25 days +7 to +14 . Ninety three percent of the patients were considered clinically cured at the end of the treatment. The most frequent bacteria isolated were Haemophilus influenzae, Streptococcus pneumoniae and Moraxella catarrhalis, and at the end of the treatment, presumed bacteriological eradication was observed in almost all patients. Adverse effects were observed in 19 of the cases, mostly mild and self limiting, including diarrhea, abdominal pain, nausea and vomiting. Treatment had to be interrupted in two cases. Gatifloxacin was found to be efficacious and safe for the treatment of acute rhinosinusitis in adults.
\end{abstract}

Key Words: Rhinossinusitis, adults, gatifloxacin.

Rhinosinusitis, an inflammatory disease, is one of the most commonly reported diseases of the sinus. It is estimated that in the United States about 35 million individuals suffer from at least one episode of acute rhinosinusitis each year. There have been reports of increasing prevalence of this disease along the years, causing many lost workdays [1].

According to periodic national physician surveys, chronic rhinosinusitis is responsible for approximately 11.6 million office visits per year. In addition, rhinosinusitis has been considered one of the five main motives for antibiotic prescriptions. In 1992, 13 million antibiotic prescriptions were made to treat acute and chronic rhinosinusitis [2].

Received on 15 September 2004; revised 18 February 2005.

Address for correspondence: Dr. Cláudia Figueiredo. Pediatric Otorhinolaryngology Division. Rua dos Otonis, 674. Vila Clementino. São Paulo - Brazil. Zip code: 04025-001. Phone: $00551155397723 .$. E-mail: claufig@uol.com.br.

The Brazilian Journal of Infectious Diseases 2005;9(2):162-168 (C) 2005 by The Brazilian Journal of Infectious Diseases and Contexto Publishing. All rights reserved.
The differential diagnosis of a viral or a bacterial infection is not always simple, although it is essential for optimum management. Antibiotics have been systematically recommended to treat bacterial sinus infections, in order to prevent both recurrences and complications, such as periorbital cellulitis, epidural and subdural empyemas, and, less frequently, osteomyelitis, meningitis, and thrombosis of the cavernous sinus [3].

Diagnosis of acute rhinosinusitis is normally based on signs and symptoms, which are usually characterized by persistent (10 to 30 days duration) nasal obstruction, headache, cough, fever, and mucopurulent discharge from the middle nasal meatus. Intense and severe initial symptoms may also indicate acute bacterial infection. The bacteria involved in the etiology of more than $70 \%$ of the cases of acute rhinosinusitis in adults and in children, worldwide, are Streptococcus pneumoniae and Haemophilus influenzae. Less frequently, Moraxella catarrhalis, Staphylococcus aureus and $\beta$ - hemolytic Streptococcus are also involved. The emergence of 
resistance and of variations in the antibiotic susceptibilities of the causative bacteria poses a great challenge to health care professionals who need to select the appropriate antibiotic [3].

Gatifloxacin is a new broad-spectrum synthetic fluoroquinolone that has proven to be safe and effective for the treatment of community-acquired respiratorytract infections. Gatifloxacin has demonstrated antibiotic activity against $S$. pneumoniae, $H$. influenzae, $M$. catarrhalis, S. aureus, Klebsiella pneumoniae, Chlamydia pneumoniae, Legionella pneumophila and Mycoplasma pneumoniae. Its half-life of eight hours permits a once a day regimen [4]. We examined the bacteriological and clinical efficacy and the safety of gatifloxacin in the treatment of non-complicated acute rhinosinusitis in adults.

\section{Material and Methods}

We report on the Brazilian data of an open-label international multicenter study, carried out in 15 centers: four in Brazil, eight in Mexico and three in Thailand. In Brazil, the study was previously submitted to and approved by the ethics committees of the four participating centers. Forty-nine adult patients with acute non-complicated bacterial rhinosinusitis were evaluated. To be eligible for enrollment in this study, subjects had to meet all of the following criteria:

- Male or female, 18 years of age or older;

- Signs and symptoms consistent with an acute maxillary sinus infection: facial pain/tenderness of one or both maxillary areas, and at least one of the following symptoms: purulent discharge from a maxillary sinus orifice, purulent discharge from the nose, or a postnasal purulent discharge;

- Radiologic confirmation of maxillary sinusitis: when at least one of the following signs were observed in one or both maxillary sinuses: opacification, visible air/fluid level, or mucosal thickening $>5 \mathrm{~mm}$.

Exclusion criteria included chronic, recurrent and complicated sinus infection, anatomic abnormalities of the nose and paranasal sinuses, history of sinus surgery, nosocomial sinus infections or infections following or associated with nasotracheal intubation, cystic fibrosis, renal insufficiency, immunodeficiency, mal-absorption syndromes, pregnancy or breast feeding, and use of antibiotics within the previous 48 hours. All female subjects received orientation regarding the importance of avoiding pregnancy and of adequate birth control.

All patients received $400 \mathrm{mg}$ gatifloxacin, once a day for 10 days. No other/concurrent antibiotic or anti-inflammatories were allowed as part of the treatment. Middle nasal meatus discharge, if present, was collected before and after treatment, under endoscopic visualization, by using a suction-collector. The material was immediately sent to the laboratory for Gram staining, culture and antimicrobial susceptibility testing.

Eligible subjects for primary efficacy analysis of the clinical response included those who had received at least five days of treatment with the study drug (at least three days for failures) and who completed the study.

The clinical response was evaluated according to the following criteria:

\section{$\underline{\text { Cure }}$}

Resolution of the acute clinical signs and symptoms relevant to the original sinus infection at the end of treatment (day +1 to day +5 ) and/or no need for antibiotic treatment at post-treatment evaluation. (day +7 to day +14$)$.

$\underline{\text { Failure }}$

Lack of improvement of signs and symptoms of the acute sinus infection after at least three days of gatifloxacin therapy.

\section{$\underline{\text { Relapse }}$}

Those patients with complete resolution of the acute signs and symptoms relevant to the original sinus infection at the end of treatment (day +1 to +5$)$, but who then developed symptoms in the post-treatment period (day +7 day to +14 ). 


\section{$\underline{\text { Unable to determine }}$}

Extenuating circumstances precluded classification as cure, failure or relapse.

Bacterial response was defined as: documented eradication, when the pathogenic bacteria isolated at the beginning of the treatment were not present at the end of the treatment; presumed eradication, when the patient presented clinical cure but a culture was not available; documented persistence, when the pathogenic bacteria were isolated at the end of the treatment; presumed persistence, when a clinical failure occurred but culture was not available; or undetermined, when the clinical parameter was undetermined.

Safety evaluation analysis was performed on all patients who had received at least one dose of the medication. It was based on the occurrence of adverse events and clinically important changes in laboratory parameters. Particular attention was given to those events that resulted in discontinuation of the study drug, or which were serious in nature. Adverse events were monitored at each follow-up visit.

Blood and urine samples were obtained for laboratory testing at the pretreatment visit, during treatment (if clinically indicated) and at the end of the treatment.

\section{Results}

Forty-nine subjects were enrolled in the Brazilian arm of the study. Most $(80 \%)$ of the patients were Caucasian, and male (65\%). Ages ranged between 18 and 68 years, with a median age of 33 .

The most frequently observed signs and symptoms of acute rhinosinusitis were purulent nasal discharge (94\%), nasal obstruction (90\%), headache (90\%), and facial pain $(71 \%)$ (Table 1$)$.

\section{Clinical efficacy}

Almost all patients presented resolution or marked improvement of their symptoms; 44 of the 49 patients were considered cured at the end of the treatment (day
+1 to day +5 ). One patient presented worsening of the symptoms, and antibiotic therapy was prescribed in another. It was not possible to determine the clinical response of four patients; two of them discontinued the medication due to adverse events and two were lost to follow-up (Table 2). Forty patients returned for the last visit (day +7 to +14 ), and 39 out of the 49 were defined as cured. The cure rate at the last followup visit was $80 \%$.

\section{Bacteriological study}

Cultures were obtained from the nasal secretion of 36 patients, and the most frequently isolated pathogenic bacteria were $H$. influenzae (16), S. pneumoniae (13) and $M$. catarrhalis (5) (Table 4). More than one type of bacteria was isolated in the cultures of 10 patients, and the most frequent association was $H$. influenzae/S. pneumoniae (four patients). Table 5 shows the relationship between clinical efficacy and the type of bacteria isolated in the inclusion visit and the high clinical cure percentage for the most frequently isolated bacteria. The results of the bacterial eradication efficacy can be observed in Table 6. Most of the bacteria that were isolated were susceptible to gatifloxacin. Presumed eradication was observed in most patients. In two cases, the analysis of the treatment efficacy was undetermined, and one patient presented failure of treatment.

\section{$\underline{\text { Adverse events }}$}

Forty-nine patients received at least one dose of gatifloxacin and were evaluated for safety. Adverse events judged as certain, probably, or possibly related to the study drug were observed in 19 out of 49 patients (39\%). They were mostly mild gastro-intestinal problems, including diarrhea in eight (16\%), abdominal pain in five $(10 \%)$, vomiting in four $(8 \%)$ and nausea in four $(8 \%)$ (Table 3). Medication was discontinued in two cases; one patient experienced a severe allergic reaction, with glottic edema on the second day of treatment, and was treated with I.V. corticosteroids; the other discontinued the treatment on the sixth day due to intense abdominal pain. Both patients experienced complete recovery. 
Table 1. Signs and symptoms of the gatifloxacin study group $(n=49)$

\begin{tabular}{lcclrr}
\hline Signs and symptoms* & $\mathbf{N}$ & $\boldsymbol{\%}$ & Signs and symptoms* & $\mathbf{N}$ & $\boldsymbol{\%}$ \\
\hline Purulent nasal discharge & 46 & 94 & Halitosis & 18 & 37 \\
Nasal congestion & 44 & 90 & Sore throat & 14 & 29 \\
Headache & 44 & 90 & Dental pain & 10 & 20 \\
Face pain & 35 & 71 & Watery nasal discharge & 9 & 18 \\
Sinus pain & 35 & 71 & Tenderness of the sinus & 7 & 14 \\
Coughing & 34 & 69 & Chills & 2 & 4 \\
Hyposmia & 31 & 63 & Epistaxis & 1 & 2 \\
Postnasal drip & 31 & 63 & Hoarseness & 1 & 2 \\
Face pressure & 28 & 57 & Ear pain & 1 & 2 \\
Malaise & 23 & 47 & Swollen sinus & 1 & 2 \\
Fever & 22 & 45 & & & \\
\hline
\end{tabular}

* A subject may have more than one sign/symptom.

Table 2. Results of clinical efficacy day +1 to +5 , end of treatment and (day +7 to +14$)$ final visit

\begin{tabular}{lrrrr}
\hline & \multicolumn{2}{c}{ V3 } & \multicolumn{2}{c}{ V4 } \\
& \multicolumn{1}{c}{ D+1/D+-5 } & \multicolumn{2}{c}{ D+7/D+14 } \\
& N & \% & N & \% \\
\hline Cure & 44 & 90 & 39 & 80 \\
Failure & 1 & 2 & 1 & 2 \\
Recurrence & - & - & - & - \\
Undetermined & 4 & 8 & 9 & 18 \\
Total & 49 & 100 & 49 & 100 \\
\hline
\end{tabular}

Table 3. Adverse events considered to have a certain, probable or possible relationship to the study drug $(n=49)$

\begin{tabular}{lrr}
\hline Adverse event & N & \% \\
\hline Any body system & 19 & 40 \\
Allergic reaction & 1 & 2 \\
Headache & 1 & 2 \\
Digestive system & 15 & 31 \\
Constipation & 1 & 2 \\
Diarrhea & 8 & 16 \\
Flatulence & 1 & 2 \\
Nausea & 4 & 8 \\
Abdominal pain & 5 & 10 \\
Vomiting & 4 & 8 \\
Nervous system & 5 & 10 \\
Dizziness & 3 & 6 \\
\hline
\end{tabular}


Table 4. Type of bacteria isolated before treatment $($ day $+1 / \mathrm{D}+5)$

\begin{tabular}{lrr}
\hline Bacteria & N & \% \\
\hline H. influenzae & 16 & 36 \\
S. pneumoniae & 13 & 30 \\
M. catarrhalis & 5 & 12 \\
S. aureus & 3 & 7 \\
E. coli & 1 & 2 \\
E. aerogenes & 1 & 2 \\
Proteus retigeri & 1 & 2 \\
Neisseria spp. & 1 & 2 \\
\hline Total & $\mathbf{4 4}$ & $\mathbf{1 0 0}$ \\
\hline
\end{tabular}

Table 5. Relationship between clinical efficacy and type of isolated bacteria before treatment (day +1 to day +5$)$

\begin{tabular}{lcccccc}
\hline & \multicolumn{2}{c}{ Cure } & \multicolumn{2}{c}{ Failure } & \multicolumn{2}{c}{ Undetermined } \\
& $\mathbf{N}$ & $\mathbf{\%}$ & $\mathbf{N}$ & $\mathbf{\%}$ & $\mathbf{N}$ & $\boldsymbol{\%}$ \\
\hline H. influenzae & $15 / 16$ & 94 & $0 / 16$ & 0 & $1 / 16$ & 6 \\
S. pneumoniae & $12 / 13$ & 92 & $1 / 13$ & 8 & $0 / 13$ & 0 \\
M. catarrhalis & $5 / 5$ & 100 & $0 / 5$ & 0 & $0 / 5$ & 0 \\
S. aureus & $2 / 3$ & 67 & $0 / 3$ & 0 & $1 / 3$ & 33 \\
Streptococcus spp. & $1 / 1$ & 100 & $0 / 1$ & 0 & $0 / 1$ & 0 \\
S. pyogenes & $2 / 2$ & 100 & $0 / 2$ & 0 & $0 / 2$ & 0 \\
E. coli & $1 / 1$ & 100 & $0 / 1$ & 0 & $0 / 1$ & 0 \\
E. aerogenes & $1 / 1$ & 100 & $0 / 1$ & 0 & $0 / 1$ & 0 \\
Proteus retigeri & $1 / 1$ & 100 & $0 / 1$ & 0 & $0 / 1$ & 0 \\
Neisseria spp. & $1 / 1$ & 100 & $0 / 1$ & 0 & $0 / 1$ & 0 \\
\hline
\end{tabular}

Table 6. Results of bacteriological efficacy according to the isolated bacteria

\begin{tabular}{lcccccc}
\hline & $\begin{array}{c}\text { Documented } \\
\text { Eradication }\end{array}$ & \multicolumn{2}{c}{$\begin{array}{c}\text { Presumed } \\
\text { Eradication }\end{array}$} & \multicolumn{2}{c}{$\begin{array}{c}\text { Presumed } \\
\text { Persistence }\end{array}$} \\
\hline H. influenzae & $\mathbf{N}$ & $\%$ & $\mathbf{N}$ & $\mathbf{\%}$ & $\mathbf{N}$ & $\mathbf{\%}$ \\
\hline S. pneumoniae & $1 / 16$ & 6 & $15 / 16$ & 94 & $0 / 16$ & 0 \\
M. catarrhalis & $0 / 13$ & 0 & $12 / 13$ & 92 & $1 / 13$ & 8 \\
S. aureus & $0 / 5$ & 0 & $5 / 5$ & 100 & $0 / 5$ & 0 \\
S. pyogenes & $0 / 3$ & 0 & $2 / 3$ & 67 & $0 / 3$ & 0 \\
Streptococcus $\mathrm{sp}$ & $0 / 2$ & 0 & $2 / 2$ & 100 & $0 / 2$ & 0 \\
E. coli & $0 / 1$ & 0 & $1 / 1$ & 100 & $0 / 1$ & 0 \\
E. aerogenes & $0 / 1$ & 0 & $1 / 1$ & 100 & $0 / 1$ & 0 \\
Proteus retigeri & $0 / 1$ & 0 & $1 / 1$ & 100 & $0 / 1$ & 0 \\
Neisseria spp. & $0 / 1$ & 0 & $1 / 1$ & 100 & $0 / 1$ & 0 \\
\hline
\end{tabular}




\section{Discussion}

The diagnosis of rhinosinusitis is often presumptive, and consequently treatment is empirical. The emergence of resistant causative bacteria is a worldwide concern and poses a great challenge for selecting effective antibiotics. Although a large percentage of patients with sinusitis are known to recover spontaneously, antibiotics have been systematically recommended in the treatment of confirmed cases of bacterial rhinosinusitis. Currently, a large selection of antibiotics is commercially available and indicated by international guidelines for the treatment of acute bacterial rhinosinusitis [1]. International guidelines indicate, amoxicillin, amoxicillin-clavulanate, cefaclor, azithromycin, clarithromycin, axetilcefuroxime, cefprozil, cefpodoxime-proxetil, levofloxacin, moxifloxacin and gatifloxacin [3].

The dilemma facing many physicians is how to select the most appropriate antibiotic from a variety of pharmacological options. According to the American Academy of Otolaryngology Committee, which is responsible for rhinosinusitis recommendations, several criteria should be considered; previous clinical trial results, regional differences in resistance patterns, antimicrobial activity against resistant pathogens, dosing facility, adverse effects, history of allergy, and cost [1].

Considering all these factors, gatifloxacin has shown to be a very attractive choice for the treatment of acute bacterial rhinosinusitis in adults. Gatifloxacin clinical efficacy has been demonstrated in many studies and clinical trials, and it has shown excellent in vitro activity against the most frequent respiratory tract infection pathogens, including penicillin-resistant bacteria, such as $S$. pneumoniae [5,6].

Gatifloxacin allows an easy to schedule dosage. Most of the reported adverse events are mild, and costs are similar to that of other antibiotics recommended for the treatment of rhinosinusitis.

Gatifloxacin, provided once a day for 10 days, gave clinical and bacteriological efficacy. Although ours was not a comparative study, other studies have reported similar results. A multicenter-randomized double blind comparative study between gatifloxacin and clarithromycin for the treatment of acute maxillary bacterial rhinosinusitis indicated similar clinical efficacy of the two drugs and better tolerability of gatifloxacin [7]. Another study comparing gatifloxacin and amoxicillin-clavulanate, as treatments for acute rhinosinusitis, demonstrated equivalent bacterial efficacy against $H$. influenzae, S. pneumoniae, and $S$. pyogenes, and superior efficacy against $M$. catarrhalis and S. aureus [8].

Several international epidemiological surveillance studies, including the SENTRY antimicrobial surveillance program, in which Brazil has had a large participation, have consistently demonstrated an annually-increasing rate of infection with isolates of $S$. pneumoniae that have diminished susceptibility to penicillin, cephalosporins and macrolides [9]. However, susceptibility to gatifloxacin among these isolates has been $100 \%$, including highly penicillin-resistant $S$. pneumoniae. All strains of $H$. influenzae and $M$. catarrhalis have also been found to be susceptible to gatifloxacin, proving it to be more efficient than amoxicillin/clavulanate and other antibiotics [10].

Only one serious adverse event was observed, leading to interruption of the treatment. The absence of photo-sensitivity is an important point, since this is a relatively common adverse effect reported for other fluorquinolones, such as moxifloxacin [11,12]. Our study confirms other previous trials, indicating that gatifloxacin is well tolerated, since most of the adverse effects were mild, essentially involving gastric-enteric disturbances .

In conclusion, the efficacy and safety profile that we found for gatifloxacin supports our recommendation of this antibiotic as an appropriate option for the treatment of bacterial rhinosinusitis in adults.

\section{References}

1. Anon J.B. Report of the rhinosinusitis task force committee meeting. Otol Head Neck Surg 1997; 117(3): S1-S48.

2. Kaliner M.A., Osguthorpe J.D., Fireman P., et al. Sinusitis: bench to bedside. Current findings, future directions. J Allergy Clin Immunol 1997;99(6):S829-48. 
3. Benninger M.S., Anon J., Mabry R.L. The medical management of rhinosinusitis. Otol Head Neck Surg 1997;117:S41-S9.

4. Bryskier A. Fluoroquinolones: mechanism of action and resistance. Int J Antimicrob Agents 1993;2(3):151-84.

5. Jones R.N., Biendenbach D.J., Erwin M.E., et al. Quality control study group. Activity of gatifloxacin against Haemophilus influenzae and Moraxella catarhalis, including susceptibility test development, E-test comparisons, and quality control guidelines for $H$. influenzae. J. Clin Microbiol 1999;37:1999-2002.

6. Lopez Sisniega J.A., Jones R.W., Kaminszczik G., et al. Gatifloxacin in the treatment of acute, uncomplicated bacterial sinusitis: an open-label, multicenter, noncomparative study. J Resp Dis 1999; 20(11):S11-S6.

7. Fogarty C., McAdoo M., Paster R.Z., et al. Gatifloxacin vs. claritromycin in the treatment of acute uncomplicated bacterial sinusitis: a randomized, double-blind, multicenter, phase III trial. J Resp Dis 1999;20(11):S17S22.

8. Bauernfeind A. Ankel-Fuchs D. Bacteriological efficacy of gatifloxacin in community-acquired respiratory infections. Antiinfective Drugs and Chemotherapy 2000; $17: 88$ (plus poster), $\mathrm{n}^{\circ} 1$.

9. Sader H.S., Gales A.C., Zoccoli C., et al. Prevalence of antimicrobial resistance among respiratory tract isolates in Brazil: 1997 and 1998 results from the SENTRY Antimicrobial Surveillance Program. Presented at the IX International Congress of Infectious Diseases. Buenos Aires, April, 2000.

10. Nicodemo A.C., Mendes C.M.F., Oplustil C.P., Sinto S. In vitro activity of gatifloxacin and nine other antibiotics against Streptococcus pneumoniae. Presented at the IX International Congress of Infectious Diseases. Buenos Aires, April, 2000.

11. Antimicrobial Treatment Guidelines for Acute Bacterial Rhinossinusitis. Otololaryngol Head Neck Surg 2000;123(1):S1-S32.

12. Brook I., Gooch W.M., Jenkins S.G., et al. Medical management of acute bacterial sinusitis recommendations of the clinical advisory committee on pediatric and adult sinusitis. Ann Otol Rhinol Laryngol 2000;119(5)suppl 182. 